Recepción: 13 / 03 / 2018

Aceptación: 14 / 05 / 2018

Publicación: 01 / 08 / 2018

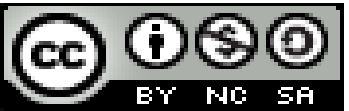

Ciencias económicas y empresariales

Artículo de investigación

\title{
La informalidad empresarial, evolución literaria que denota un fenómeno complejo
}

Business informality, literary evolution that denotes a complex phenomenon

A informalidade de negócios, evolução literária que denota um fenômeno complexo

Julián R. Rodríguez-Soto ${ }^{\mathrm{I}}$

julian.rodriguez@campusucc.edu.co

Carlos Dussán-Pulecio II

carlos.dussanpu@campusucc.edu.co

Correspondencia: julian.rodriguez@ campusucc.edu.co

\footnotetext{
${ }^{\text {I } D o c e n t e, ~ U n i v e r s i d a d ~ d e ~ M e d e l l i ́ n, ~ M e d e l l i ́ n, ~ C o l o m b i a . ~}$

II Docente, Universidad Cooperativa de Colombia, Ibagué/Espinal, Colombia.
} 


\section{Resumen}

El artículo tiene como objetivo analizar la literatura existente y la evolución de las investigaciones relacionadas con la informalidad empresarial, utilizando la Revisión Sistemática de Literatura, para determinar las posibles causas que ocasionan dicho fenómeno. Para tal finalidad se realizó una búsqueda de artículos a partir del término "Informalidad Empresarial" o "Informal Sector", en los Journal y revistas indexadas que publican resultados o avances de investigación, en las bases de datos Scopus, e incluso fueron consultados algunos informes emitidos por agremiaciones e instituciones de orden estatal. La búsqueda se focalizó en países que guardan similitud con Colombia, en cuanto al número de habitantes y en fenómenos de empleo. Los principales hallazgos del estudio bibliométrico dan cuenta del interés sobre el tema específicamente desde los años 90, además conceptualizan la informalidad empresarial como la imposibilidad de las empresas en generar empleo formal, aunque este no es el único enfoque encontrado; es recurrente encontrar investigaciones que aun coincidiendo con el postulado anterior están agrupadas en criterios gerenciales como: "Factores Sociodemográficos", "Ley, Ordenanzas y Gobernabilidad" y "Macro Indicadores de visibilidad Exterior". En conclusión, son escasas las publicaciones en revistas de alto impacto, sin embargo, la tendencia ha sido creciente y existen diferentes perspectivas interesantes para futuras investigaciones.

Palabras clave: legislaturas; empresa; desempleo; sector informal; políticas públicas.

\section{Abstract}

The physical, verbal and psychological aggressions are known as gender violence, they were integrated into the Comprehensive Organic Penal Code in 2014 and their results have a drastic influence on society. The purpose of this study was to determine what is the criminal responsibility of the procedural subject in the types of gender violence in Ecuador. The sample of the study, to determine what has been the incidence and penal impact on the person processed, was confirmed by the cases of sexual abuse against women in 2014. These data were exposed by the National Institute of Statistics and Census. The following variables were determined: number of cases that have been reported and those that have been sanctioned. The findings reveal that the creation of instances such as police stations for women and families generated a greater number of complaints. Although the number of complaints has increased, it does not reveal a real percentage of the current situation due to the lack of knowledge of society about this crime, its 
causes and its sanction. It continues to reflect a high rate of impunity for the multiple social obstacles and social pressure.

Keywords: gender violence; women; physical aggressions; Ecuador.

\section{Resumo}

O artigo tem como objetivo analisar a evolução da literatura e investigação relacionada com a informalidade do negócio, utilizando a Revisão sistemática de literatura para determinar as possíveis causas que causam este fenômeno. Para este fim, uma pesquisa foi realizada a partir do termo "informalidade empresarial" ou "setor informal", no Jornal e revistas que publicam resultados ou progresso da pesquisa, com base em dados Scopus indexado, e foram mesmo consultou alguns relatórios emitidos por associações e instituições de ordem estatal. A pesquisa foi focada em países que são semelhantes à Colômbia, em termos de número de habitantes e fenômenos de emprego. As principais conclusões de o estudo bibliométrico perceber o interesse sobre o assunto especificamente da década de 90, também conceituar informalidade negócios como a incapacidade das empresas de geração de emprego formal, embora esta não é a única abordagem encontrada; Recorre encontrar pesquisas que mesmo coincidindo com o postulado anterior são agrupados em critérios de gestão, tais como: "fatores sociodemográficos", "Direito, portarias e Governação" e "Macro visibilidade Indicadores Exterior". Em conclusão, há poucas publicações em periódicos de alto impacto, no entanto, a tendência vem crescendo e existem diferentes perspectivas interessantes para pesquisas futuras.

Palavras chave: legislaturas; empresa; desemprego; setor informal; políticas públicas.

\section{Introducción}

El fenómeno de la informalidad empresarial en el mundo y Latinoamérica no es tan nuevo como parece y está asociado a múltiples características según un Informe del Banco Mundial (Banco Mundial, 2009). Algunos teóricos lo asocian al ser individual que busca la maximización de sus expectativas. De hecho, desde 1934 estudios documentados sobre la teoría económica y el desarrollo determinaron que el ser humano busca la maximización de sus utilidades (Schumpeter, 1934), aunque dicha teoría había sido abordada desde un siglo atrás por Adam Smith y David Ricardo. En este sentido, Bobrow \& Dryzek (2000) afirman:

Tomando como supuesto que el sujeto actúa de manera racional en pro de su interés particular, no hay políticas que puedan beneficiar al total de la población ocupada en el sector informal, y por 
lo tanto, el tomador de decisiones debe enfrentarse con un conflicto de intereses entre distintos grupos de coacción (o neighborhoods) en los que una determinada política puede resultar benéfica para unos pero perjudicial para otros, teniendo así que prever las consecuencias colectivas de su implementación bajo diferentes circunstancias (p. 157).

Aunque es muy poca la literatura especializada que se encuentra sobre el origen de la informalidad empresarial, los autores hallados coinciden en afirmar que el origen de este fenómeno se encuentra relacionado con el impacto de la industrialización y los procesos de producción en línea y a gran escala. En este sentido, es oportuna la opinión de (Townsend 2000, p, 377), cuando afirma que: “[...] debido al cambio de una economía de manufactura a una economía de información, la naturaleza del trabajo ha sido alterada y el ambiente laboral y empresarial está bajo modificaciones dramáticas". En muchos casos, la automatización ha traído como consecuencia la desocupación, corroborando que las modificaciones en el mundo del trabajo son el resultado de los cambios en la empresa y por ende de la globalización económica (Mejía, 2006).

También es evidente que la dificultad de los países en vía de desarrollo para participar en las economías de escala ha restado importancia a la misma empleabilidad, por lo que la nueva realidad mundial en temas de empleo hace hincapié en que la mano de obra dejó de ser una demanda potencial para convertirse en un costo de producción, costo que tiene que ser el menor posible para aumentar la rentabilidad del negocio (López, 2008).

El presente trabajo indaga acerca del impacto negativo, derivadas de los altos índices de informalidad empresarial en los países objetos de la revisión sistemática de literatura; por tanto, el objetivo en esta revisión sistemática de literatura busca determinar las diferentes aristas de como la comunidad científica y académica ha investigado la informalidad empresarial, específicamente en Latinoamérica.

En países de Latinoamérica, y desde luego en Colombia, es muy notorio que las empresas hayan disminuido su actividad productiva e industrial que requiere mayor mano de obra, dando paso a actividades más comerciales.

Lo que sí es muy recurrente es la forma como en América Latina y Colombia surgió la empresa, la empresarialidad, los sectores económicos y, en ese mismo orden, el empleo. (Parra, Catica, \& López, 2015). 
El primer sector económico que apareció en Latinoamérica fue el sector agrícola, y desde ahí se originaron las relaciones de empleo. Este sector, pese a ser el más antiguo, no ha logrado ser gran impulsor de la economía, a excepción de Brasil y Argentina donde se ubica en un renglón preponderante de la economía actual, aunque sin ser el primero. (Paz, 2013).

Otro asunto que influenció en la creación de empresas y la conformación de un tejido empresarial privado (sin influencia o dominio estatal), fueron las profundas transformaciones que han experimentado en su entorno social, político y económico los países de Latinoamérica, esto sin duda logró no solo incentivar la creación de empresas sino además marcar las tendencias de las relaciones laborales desde principios de los años sesenta y decenios posteriores.

Algunos de los cambios más drásticos y de mayor repercusión se originaron con la crisis de los sistemas políticos en numerosos países, a partir de 1963; por el golpe militar de Honduras, seguido de Brasil y Bolivia (1964), Argentina (1966), Perú y Panamá (1968) Ecuador (1973), Chile y Uruguay (1973). (Bronstein, 1995).

Es válido mencionar que desde la Colonia hasta los inicios de las décadas de los 70 en Latinoamérica el mayor empleador y propietario de los medios y fuentes de producción ha sido el Estado. Con el final de las dictaduras militares y el efecto democratizador, que dio por terminado los regímenes dictatoriales, también se desescalaron las limitaciones públicas, el derecho a la sindicalización y las relaciones colectivas del trabajo que se encontraban suprimidas.

Pero el modelo político de la democracia no fue suficiente para reactivar la economía y. era claro que el modelo proteccionista e intervencionista del Estado mostraba grandes signos de agotamiento; La elevada inflación y la ineficiencia de los Estados para generar empleo conllevaron a la implementación de una nueva estrategia neoliberal por medio de los llamados programas de ajuste estructural. El país pionero en la implantación de dicha estrategia fue Chile, y el éxito económico alcanzado por este país motivó a aplicar dicha estrategia en la década de los 80 en países como México, Costa Rica y Bolivia, seguidos de Perú, Colombia y Venezuela, al inicio de los 90.

Dentro de las principales características contempladas por la estrategia neoliberal se pueden mencionar: la disminución del papel del Estado, el recorte del gasto público, las aperturas económicas hacia mercados más abiertos y menos protegidos y la reasignación de recursos necesarios por parte del Estado para incentivar el aparato productivo (Salazar Borrego, 2000). 
Por lo anterior, se puede evidenciar que la creación de empresa, la cultura emprendedora y el desarrollo empresarial en Latinoamérica datan de finales del siglo XVIII y principios del siglo XIX. En el caso de Colombia tiene origen a principios del siglo XIX. En sus inicios el desarrollo empresarial se fundamentó en la agricultura, las empresas que surgieron entre los años 1900 1940, como la Compañía Nacional de Chocolates S.A., hoy comercial Nutresa y la empresa de bebidas colombiana, hoy Postobón, pese a que en sus inicios tuvieron una alta dependencia del sector agrícola, hoy se mantiene como un referente importante de la economía actual.

Estas particularidades en la taxonomía empresarial de Latinoamérica se han mantenido vigentes. Cuando se analiza la historia económica de Colombia en las últimas décadas, son evidentes dos aspectos. En primer lugar, no hay nombres nuevos entre las empresas más grandes del país, con excepción de las de capital extranjero. En segundo lugar, no hay exportaciones nuevas exitosas entre los principales productos nacionales, e incluso los productos de exportación que se mantienen vigentes datan de varias décadas atrás y no hay mucho que destacar en términos de valor agregado. (Cruz, 2009). Por lo que es pertinente afirmar que la estructura empresarial del país y de Latinoamérica está conformada por las Micro Pequeñas y Medianas Empresa, Mipymes mayoritariamente.

Con la intención de determinar la evolución literaria del concepto de informalidad laboral, el presente artículo se estructura de la siguiente manera: primero, un marco conceptual que incluye los factores preponderantes de la creación de empresas en algunos países de Latinoamérica y la representatividad de la microempresa y pequeña empresa en el Continente. Segundo, principales hallazgos y resultados derivados del análisis cuantitativo y cualitativo, finalmente las conclusiones derivadas de la revisión sistemática de literatura.

\section{Importancia del problema}

\section{Factores preponderantes de la creación de empresas en países de América Latina.}

Cuando se indaga por la creación de empresas en América Latina, o acerca del fenómeno de la informalidad, se encuentra una gran similitud con el concepto de emprendimiento, o entrepreneurship. Al respecto Álvarez (2011) señala:

Desde una perspectiva muy general, podría decirse que las investigaciones en este campo se basan en tres grandes enfoques. Por una parte, el enfoque económico, donde se imponen los aspectos relativos a la racionalidad económica y, a grandes rasgos, se plantea que el 
emprendimiento obedece a cuestiones puramente económicas (Audretsch y Keilbach, 2004; Audretsch y Thurik, 2001; Parker, 2004; Wennekers et al., 2005, entre otros). Por otra parte, el enfoque psicológico postula que son los factores individuales o los rasgos psicológicos de las personas los que determinan la actividad emprendedora (Carsrud y Johnson, 1989; Collins, Moore y Unwalla, 1964; McClelland, 1961, entre otros). Por último, el enfoque sociológico o institucional defiende que el entorno socio cultural condiciona la decisión de crear una empresa (Aldrich y Zimmer, 1986; Berger, 1991; Busenitz, Gomez y Spencer, 2000Manolova, Eunni y Gyoshev, 2008; Shapero y Sokol 1982; Steyaert y Katz, 2004, entre otros) (p.18).

Lo que sí es evidente es que el emprendimiento planteado o entendido desde cualquiera de los enfoques mencionados anteriormente (económicos, psicológicos, sociológica-institucional) ha sido uno de los grandes impulsores de la consolidación del tejido empresarial en todos los países de Latinoamérica; por tanto, casi todos los autores consultados, y que son referente en la construcción de este documento, convergen en las mismas posiciones: consideran que la micro empresarialidad, o mi pymes, representa una parte importante de la estructura económica en la mayoría de los países (Mungaray, Ramírez, Aguilar , \& Beltrán , 2007), y la existencia de dichas unidades productivas son comunes en las regiones en vía de desarrollo, desde luego Latinoamérica es una de ella (Rodríguez Soto \& Hernández Sanchez, 2013).

A continuación, se enuncian los aspectos positivos y negativos de la consolidación empresarial basada en el emprendimiento.

\section{Cuadro 1}

Aspectos positivos y negativos de la consolidación empresarial basada en el emprendimiento

\begin{tabular}{|c|c|}
\hline POSITIVOS & NEGATIVOS \\
\hline Fortalecimiento del tejido empresarial & Baja Productividad y Competitividad \\
\hline $\begin{array}{l}\text { Descentralización del Estado como } \\
\text { generador de empleo }\end{array}$ & Regímenes tributarios diferenciales \\
\hline Dinamismo Económico & Intormalidad Empresarial \\
\hline Flexibilización Laboral & Trabajo Informal \\
\hline
\end{tabular}

Fuente: Elaboración propia, a partir del informe del Banco Interamericano de Desarrollo (2010)

Para efectos de desarrollo de este documento, y facilitar la búsqueda de referencias, la investigación se centró en la informalidad empresarial y el trabajo informal como posible causa 
de una estructura empresarial fundamentada en las Mipymes y producto de una excesiva política de estímulo al emprendimiento.

\section{La representatividad en la microempresa y pequeña empresa en América Latina.}

Son muchos los autores que han logrado compilar datos sobre la composición empresarial en América Latina (Bonilla, 2003; Mungaray, Ramírez, Aguilar, \& Beltrán, 2007; Arruñada, 2007; Santamaría y Rozo, 2009; Quevedo y Ramírez, 2012; Fedesarrollo, 2013; Rodríguez y Hernández, 2014). De hecho, van desde fuentes documentales reconocidas hasta estudios liderados por agremiaciones y distintos entes estatales o de economía mixta en los diferentes países. Los datos son interesantes y llaman mucho la atención por la gran preponderancia que representan estas unidades productivas (micros y pequeñas), para las economías de los países de América Latina.

A continuación, se analiza el impacto de la micro y pequeña empresa en la economía de algunos países de América Latina y la contribución en el desarrollo local y regional de acuerdo con el país de origen y su PIB. Al respeto Aguilar Barceló, Texis Flores; \& Ramirez Angulo (2007) presentan datos sobre la importancia que representan las microempresas. Dicha información se encuentra documenta en el artículo: El poder del mercado en empresas de Bajo California:

La microempresa representa una parte importante de la estructura económica en la mayoría de los países (Mungaray, Ramírez, Aguilar y Beltrán, 2007); muchas de estas surgen del aprovechamiento de las brechas dejadas por el gran capital para sacar provecho de una pequeña oportunidad de negocio, debido a su tamaño y flexibilidad (Max-Neef, 1998).

Si bien es cierto que muchas micro empresas operan bajo el resguardo de la formalidad, entendida como el registro de sus actividades ante la autoridad correspondiente, existen estimaciones de que al menos el 20\% del PIB de las Economías latinoamericanas podrían estar siendo generado exclusivamente por las microempresas en el sector informal (Bacchetta, Ernst y Bustamante, 2009) (p.64).

De acuerdo con cifras del Banco Mundial (2007), las empresas informales (aquellas que no pagan impuestos ni cuentan con un registro mercantil) y los trabajadores por cuenta propia no calificados representan cerca del 60\% de la fuerza laboral de los países emergentes. Este hecho, unido a los bajos niveles de productividad de las empresas informales y su asociación con economías de base estrecha, hace que la empresarialidad informal se muestre hoy como uno de 
los grandes retos en materia de política pública económica y social en América Latina (Banco Mundial, 2007).

Otra referencia importante, es que, pese a que el sector empresarial informal ha sido considerado un sector marginal o residual en términos de contribución a la economía, hay evidencias de que el crecimiento de la informalidad empresarial, como en el caso de Ecuador, no es para nada despreciable para el Estado, puesto que ha sido capaz de minimizar problemas estructurales de la economía y generar empleo (Torresano \& Díaz Chistiansen, 2014).

En Colombia se considera que la sobre exposición de la cultura emprendedora en el país ha generado un desbordado crecimiento de las micro, pequeñas y medianas empresas. En el país, Bogotá se caracteriza por tener una dinámica empresarial positiva: cada año se crean en promedio 68 mil empresas y se renuevan cerca de 255 mil. En el 2012, el número de empresas localizadas en Bogotá llegó a 337 mil empresas. En este mismo año se incrementó en $0.3 \%$ el número de empresas creadas con respecto a 2011 y se alcanzó la mayor tasa de creación de empresas de los últimos 4 años. El crecimiento en la creación de empresas fue promovido por las políticas gubernamentales de fomento al emprendimiento y fortalecimiento de las Mipymes. (Sociedades, 2013). Otro dato importante del informe Regional Bogotá 2009-2012, es la estructura empresarial en Bogotá, donde se evidencia que las microempresas representan un $87 \%$, las pequeñas empresas representan un 9\%, las medianas empresas un 3\% y las grandes empresas representan tan solo un 1\%, estos datos aportados por el informe en el 2012 entregado por la Cámara de Comercio de Bogotá. Estos datos dan cuenta de la dinámica y la importancia que representan las Mipymes para la economía del país, como se puede evidenciar en el siguiente Gráfico No. 1.

Grafico 1. Estructura Empresarial Bogotá \& Cundinamarca

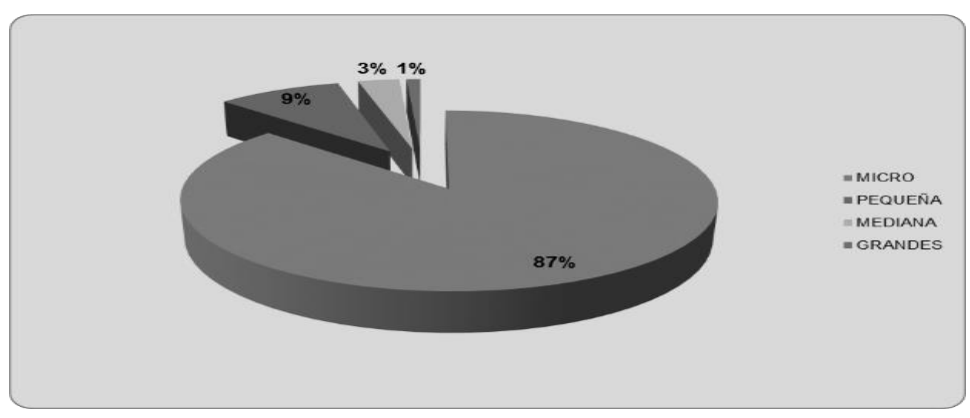

Fuente: elaboración propia, a partir del Informe Económico de la Cámara de Comercio de Bogotá (2009-2012) 
Adicional a lo anterior, las micro, pequeñas y medianas empresas cada vez tienen más peso en las economías de los países en vías de desarrollo o en economías emergentes, debido al impacto directo que este grupo empresarial tiene, tanto en la generación de empleo como en su representación en el PIB. Para el caso de Colombia las Mipymes representan cerca de un 90\% del total de las empresas y generan entre un 60\% y 70\% del empleo (Espinosa Romero, 2013).

Otro caso destacado es el de Bolivia, donde las microempresas y el trabajo por cuenta propia representan cerca del $80 \%$ de la población económicamente activa y aportan cerca del $20 \%$ del Producto Interno Bruto del país; de dichos porcentajes, el 63\% se ubica en el sector informal. (Parra Bernal, 2013).

Todo lo anterior denota la importancia de la Mipymes en los países en vía de desarrollo y en Latinoamérica, lo cual conlleva a indagar sobre las principales características de una estructura económica fundamentada en las Mipymes. En primer lugar, este tipo de organizaciones (micro, pequeñas y medianas), son comunes en los países en desarrollo, los modos de producción inherentes de estas economías responden a una transición a la modernidad (Schneider \& Enste, 2000); el sector informal actúa como amortiguador ante los continuos contrastes económicos en los países en desarrollo, es decir, se expande en las recesiones económicas o se contrae o disminuye en épocas de bonanzas. (Schneider F., 2012). Por último, las relaciones o los mecanismos fiscales ejercidos por los entes gubernamentales de cada país son determinantes, en relación con el comportamiento de la informalidad empresarial. La literatura consultada evidencia que el tamaño relativo del sector informal disminuye con el desarrollo general, aumenta con la carga de la regulación y disminuye con la fuerza de monitorización (Johnson et al., 1997; Schneider y Enste, 2000; Friedman et al., 2000; y Loayza, Oviedo y Serv'en, 2005).

\section{Metodología}

La metodología desarrollada para la construcción de este documento parte de una Revisión Sistemática de Literatura, esto desde luego conlleva varios retos. El primero, consiste en definir las ecuaciones de búsqueda. Es evidente el interés sobre el tema, de hecho aunque es escasa la información concentrada en bases de datos, los hallazgos se encuentran concentrados y documentados por entes gremiales, por ejemplo en el caso España el PATT, en Colombia Fedesarrollo, en Perú el Ministerio de Trabajo y Promoción de Empleo, entre otros, lo que hace compleja la búsqueda, por lo que se requirió definir unos criterios de interés que ayudaran a la 
selección de documentos que hicieran referencia solo a temas administrativos y gerenciales. Dichos criterios fueron: factores sociodemográficos, ley, ordenanzas y gobernabilidad y macro indicadores de visibilidad exterior. Todos estos criterios fueron clave en el refinamiento y la selección de los documentos.

Posteriormente, se continuó con la clasificación de los documentos hallados en la consulta de bases de datos especializadas (Scopus). Este estudio y análisis tuvo un carácter exploratorio sobre las investigaciones referentes al tema y cumpliendo con los criterios mencionados en el párrafo anterior. Además, el estudio exploratorio incluyó una búsqueda en los países que guardan mayor similitud con Colombia (Perú, Venezuela, Ecuador, Bolivia y México). Finalmente, es evidente un avance significativo en literatura referente al tema de la informalidad empresarial, pero muchos de estos documentos se originan o hacen citaciones de encuestas, estudios estadísticos e iniciativas investigativas lideradas por entes gremiales de cada uno de los países donde se focaliza la muestra y los datos referentes al tema.

\section{Resultados.}

\section{Resultado de la Revisión Sistemática de Literatura}

La primera discusión que enmarca el debate sobre la informalidad empresarial o sector informal, como lo acuñan la mayoría de los artículos hallados, se refiere al origen del concepto, que coincide con el informe de Kenia, de la Oficina Internacional del Trabajo OIT en 1972. Este sería como el punto de partida de donde se derivan múltiples investigaciones y abordajes sobre el tema (Osta Trestini, 2007).

Otra recurrencia originada en el proceso de búsqueda de la información es que no existe una base jurídica bien definida que proteja al trabajador informal, ni en Colombia ni en Latinoamérica; por tanto, mientras que persista esta postura frente al trabajo informal va a continuar como una opción de subsistencia para el trabajador cuenta propia, como para el empresario que pretenda mejorar sus índices de productividad a costa del mal uso de los recursos humanos. (Rubio, 2014). A partir de este punto de referencia es que se han elaborados múltiples conceptos y se han realizado investigaciones nacionales e internacionales, aunque el tema es muy importante en la búsqueda apoyada por la base de datos SCOPUS no es evidente un desarrollo del tema. Esto es explicable porque muchas de las investigaciones son ejecutados por entes gubernamentales y se focalizan en una comunidad puntual, como por ejemplo el caso de los vendedores informales de 
Guayaquil, en Ecuador (Torresano \& Díaz Chistiansen, 2014); los comerciantes de ropa usada en el Alto y la Paz - Bolivia (Parra Bernal, 2013), los tenderos y transportadores de Lima en Perú (De Soto, 1987), los comerciantes por cuenta cuanta propia en Venezuela, los microempresarios del Valle del Cauca en Colombia (Santa María \& Rozo , 2009) y los micronegociantes en el Bajo Tijuana, en México (Aguilar Barceló, Texis Flores, \& Ramirez Angulo, 2007).

Además, es importante mencionar que la mayoría de las investigaciones que centran su interés en el sector informal o la informalidad empresarial, surgen de una encuesta de hogares, como la Encuesta Anual de Hogares Urbanos (EAHU) y la Encuesta Permanente de Hogares (EPH). Este instrumento ha sido replicado en muchos países de Latinoamérica, con el fin de determinar el porcentaje de informalidad laboral y empresarial de las economías de la región (Paz, 2013; Santamaría, 2007; Schneider, 2012).

En esta investigación se utilizó la ecuación de búsqueda: "Informal Sector Latín América", resultando 67 artículos, de los cuales 42 centran su estudio en un lugar, país o región de América Latina, representando el $63 \%$ de la búsqueda; otro $22 \%$ representado en 15 artículos, centran su interés en las dificultades para la productividad y competitividad de la región, cuando se fundamenta su desarrollo en una economía informal, y el 11\% restante representado en 10 artículos, centran la discusión en las reformas o flexibilizaciones laborales que se han generado en los diferentes países de América Latina, con el fin de disminuir la informalidad empresarial o dar garantías al sector informal para que se formalicen. En la Gráfica 2 se puede evidenciar la dinámica de producción científica para el periodo 2005-2015.

\section{Gráfico 2}

Comportamiento bibliométrico de Informal Sector -Latín América (2005-2015)

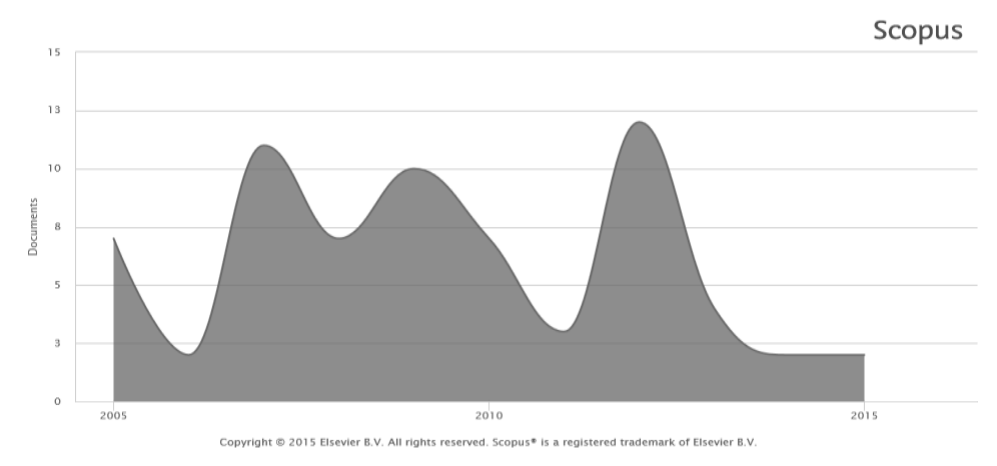

Número de publicaciones: 67

Fuente: Scoopus 2015. 
A partir de la misma ecuación de búsqueda se lograron evidenciar diversas aristas en torno al comportamiento del sector informal y el dinamismo que tiene este concepto, los diferentes significados y cómo estos avances y resultados de investigación han servido de insumo y herramienta con otras investigaciones en cada uno de los países; es decir, luego de que se presentan avances y resultados de investigación el tema logra un actividad e interés de orden estatal o gremial en los países de origen, los casos más puntuales se evidencian en España, Venezuela, Colombia y México.

Por ejemplo, en España, luego de publicado un informe sobre la estrategia de formalización adoptada en ese país, se encuentran evidencias de la poca efectividad de los denominados Puntos de Asesoramiento e Inicio de Tramitación- PAIT, algo muy similar sucedió en Colombia con los Centros de Atención Empresarial. Es evidente que las estrategias impactan de forma positiva el registro legal de la constitución empresarial, pero poco contribuyen con la generación de empleo formal; por tanto, es pertinente afirmar que dicha estrategia es válida para el proceso de registro, pero poco asertiva en formalización empresarial (Arruñada, 2007).

Otro ejemplo es el de Venezuela, donde las publicaciones con los resultados de los procesos de flexibilización laboral que pretendían disminuir la informalidad empresarial y laboral del país conllevaron a un profundo análisis por parte del ministerio público, en este país mostrando que no se lograron los resultados macro económicos perseguidos, ni la generación de empleo, ni el aumento de los índices de productividad y la dinamización del mercado laboral; así mismo, otros indicadores no lograron repuntar luego de entrar en vigencia la normatividad (Hernández Arias, 2005); por tanto, es evidente que después de que las investigaciones fueran publicadas aumentara el interés por el tema y se lideraran muchas otras por parte del Estado con el fin de afinar y reformular el proceso.

\section{Análisis Bibliométrico de los Documentos}

Utilizando la ecuación de búsqueda definida en la metología, los resultados preliminares arrojaron una gran cantidad de documentos por lo que se decidió centrar la búsqueda en aquellos países que mostraban mayores similitudes con Colombia, en relación con el tamaño poblacional, esquemas de producción, dinámica productiva y cercanía. Se tomaron como países de referencia: Venezuela, Bolivia, Ecuador, Perú, y México. 
Con respecto a la clasificación temática usada en la selección de artículos, se tuvieron en cuenta los criterios expuestos en el artículo: Causas de la informalidad en Perú, en el que el autor define tres criterios que se ajustan a la búsqueda bibliográfica con fines administrativos y gerenciales: 1. factores sociodemográficos; 2. ley, ordenanzas y gobernabilidad, 3. macro indicadores de visibilidad exterior (Loayza, 2009), como se muestra en el Cuadro 2.

\section{Cuadro 2}

Criterios de selección de artículos

\begin{tabular}{lll}
\hline \multirow{2}{*}{ Criterios } & \multicolumn{2}{l}{ Artículos } \\
\cline { 2 - 3 } & No. & $\%$ \\
\hline Factores sociodemográficos & 21 & $57 \%$ \\
\hline Ley, ordenanzas y gobernabilidad & 7 & $19 \%$ \\
\hline Macro indicadores de visibilidad exterior & 9 & $24 \%$ \\
\hline Total & & $100 \%$ \\
\hline
\end{tabular}

Fuente: Elaboración propia, a partir del estudio de Loayza (2009).

Además, se realizó un análisis exploratorio, que definió unos tópicos de interés para cada uno de los criterios de búsqueda. Esto con el fin de hacer una selección más detallada y refinar el análisis exploratorio. Lo anterior se amplía en el Cuadro N. 3.

\section{Cuadro 3}

Análisis exploratorio por tópicos de interés

\begin{tabular}{|c|c|c|c|}
\hline \multicolumn{2}{|l|}{ Temática } & \multicolumn{2}{|c|}{ Artículos } \\
\hline Criterios & Tópicos de interés & No. & $\%$ \\
\hline \multirow[t]{3}{*}{ Factores Sociodemográficos } & Empleo, subempleo y precarización laboral & 6 & $16 \%$ \\
\hline & Estudios sectoriales (región, país, sector económico) & 10 & $28 \%$ \\
\hline & Riesgos laborales y salud & 5 & $13 \%$ \\
\hline Ley, ordenanzas y gobernabilidad & Flexibilidad laboral & 7 & $19 \%$ \\
\hline \multirow{2}{*}{$\begin{array}{l}\text { Macro Indicadores de visibilidad } \\
\text { Exterior }\end{array}$} & Impacto económico global & 7 & $19 \%$ \\
\hline & Productividad y competitividad & 2 & $5 \%$ \\
\hline Total & & 37 & $100 \%$ \\
\hline
\end{tabular}

Fuente: Elaboración propia a partir de Loayza (2009) y la base de datos Scopus

Para el caso de los hallazgos en relación con los factores sociodemográficos y los principales tópicos de interés evidencian un mayor avance investigativo de la informalidad empresarial o sector informal, con un porcentaje del 57\%, seguido del criterio: macro indicadores de visibilidad exterior, que estaría en el segundo nivel de avance con un porcentaje del 24\%; finalmente, en el tercer nivel, estaría: Ley, Ordenanzas y Gobernabilidad con una representatividad del 19\%. 


\section{Conclusiones}

La primera gran conclusión es que pese a la alta recurrencia de este fenómeno y al interés de los entes estatales por abordar e investigar este tema no existe un concepto generalizado que permita definir el sector informal o informalidad empresarial, aunque todos los investigadores usan como guías los pronunciamientos dados por la Organización Internacional del Trabajo (OIT), y su influencia en las políticas implantadas por los gobiernos de América Latina.

Las principales categorías en que se divide el empleo informal generado por la informalidad empresarial, sirven como muestra o insumo a más de un $89 \%$ de los documentos publicados. Según las investigaciones realizadas, y a partir de la revisión sistemática de literatura, son: trabajadores por cuenta propia dueños de sus propias empresas del sector informal; empleados dueños de sus propias empresas del sector informal; trabajadores familiares, auxiliares independientes, vinculados a empresas del sector formal o informal; miembros de cooperativas de productores informales; trabajadores por cuenta propia que producen bienes exclusivamente para el propio uso final de su hogar; asalariados que tienen empleos informales (Organización Internacional del Trabajo (OIT), 2003).

Pese a los esfuerzos tributarios y normativos emprendidos por muchos gobiernos, son pocos los avances en la disminución de los índices de informalidad empresarial. Al respecto la Comisión Económica para América Latina y el Caribe, CEPAL, complementa que:

Los países de América Latina han buscado por medio de una serie de reformas tributarias, laborales y económicas combatir las crisis en el mercado laboral causadas por la alta informalidad empresarial, sin embargo, los sistemas de protección social de la región están lejos de ser inclusivos y además presentan brechas normativas que los hacen vulnerables. (CEPAL, 2012).

Otra conclusión, que surge de la revisión sistemática de literatura es que en Latinoamérica es común iniciar un proyecto empresarial al margen de los requisitos, o sea en la informalidad empresarial. La necesidad de emprender para el sustento diario del emprendedor y su núcleo familiar es otra característica recurrente en la búsqueda de documentos y resultados de investigación, y es mayor la incidencia en regiones distantes de los polos de desarrollo o ciudades capitales. 
Por consiguiente, es notorio el interés y los esfuerzos por coadyuvar al naciente empresario a que se consolide en una empresa capaz de atender sus obligaciones tributarias, legales y laborales que su objeto social requiere. Estos esfuerzos se ven traducidos en normativas, estímulos y beneficios que causan un gran impacto al fisco de la nación, pero no son directamente proporcionales con el retorno de dichas empresas; por tanto, muchas investigaciones revisadas dan cuenta que el empresario hace aprovechamiento del beneficio, pero considera que es bajo el retorno al momento de formalizar su unidad productiva, frente a los altos costos tributarios y legales de los países objeto de este estudio.

De acuerdo con la revisión literaria se hace pertinente nuevas investigaciones que caractericen el fenómeno desde la estructura empresarial de estas organizaciones, micro y pequeñas; con el fin de determinar cuáles son sus potencialidades de crecimiento y como estas organizaciones pueden contribuir con el desarrollo y el crecimiento económico de la región.

Por último, la reducción de la informalidad empresarial no consiste solo en registrar las operaciones tributarias y obtener registros, es necesarios que las unidades productivas crezcan en tamaño sus trabajadores sean afiliados a los sistemas de seguridad social; es decir, que toda empresa opere con contratos de trabajo decente y con protección social.

\section{Referencias Bibliográficas}

Aguilar Barceló, J. G., Texis Flores, M., \& Ramirez Angulo, N. (2007). Conformación de la expectativa de formalización de la microempresa marginada en México. Innovar, pp. 63-73.

Álvarez, C. (2011). Una Década de Investigación basada en el GEM: Logros y Retos. Revista Latinoamérica de Administración, pp. 16-37.

Arruñada, B. (2007). El Imperativo de Eficiencia en la Formalización Empresarial. Revista Globalización, Competitividad y Gobernabilidad, pp. 106-115.

Banco Interamericano de Desarrollo. (2010). La era de la productividad. México D.F.: Carmen Pagés.

Banco Interamericano de Desarrollo. (2011). La Era de la Productividad. México D.F: Carmen Pages.

Banco Mundial. (2007). Informality in LAC - ABarrier to growth and social Welfare. Washington: World Bank Publications.

Banco Mundial. (2009). Informe anual (Flagship). Washington: Banco Mundial. 
Bobrow, D., \& Dryzek, J. (2000). Policy Analysis by Design. Pittsburgh: University Pittsburgh Press.

Bronstein, A. (1995). Cambios sociales y relaciones de trabajo en América Latina: Balances y Perspectivas. Revista Internacional del Trabajo, pp. 185-209.

Castro G, O. E., \& Rodríguez S., J. R. (2015). La situación actual del trabajo infantil en el Espinal-Tolima: Revista Virtual Universidad Católica del Norte, pp. 258-269.

CEPAL. (20012). Proyecto de programa de trabajo del sistema de la CEPAL, 2014-2015. Trigésimo periodo de secciones de la CEPAL. San Salvador: Original Español.

Cruz, H. A. (2009). Empresarialidad y Transformación Productiva. IGNIS, p.15.

De Soto, H. (1987). El Otro Sendero la Revolución Informal. Lima Perú: Oveja Negra.

Espinosa Romero, F. (2013). Alcances y limitaciones de los modelos de capacidad predictiva en el análisis del fracaso empresarial. AD- minister, pp. 23-39.

Hernández Arias, A. (2005). Flexibilización y organización del trabajo. Revista de Ciencias Sociales, pp. 256-276.

Loayza, N. (2009). Causas y Consecuencias de la informalidad en Perú. Estudios Económicos, pp. 43-64.

López, C. (2008). Proyecto de Ley de Transformación Social. Congreso de la Republica. Bogotá, Colombia: Congreso de la Republica.

Mejía J., M. R. (2006). Educación(es) en la(s) globalización(es) I. Bogotá D.C: Desde Abajo. Mungaray, A., Ramirez, N., Aguilar, J., \& Beltrán, J. (2007). Poder de mercado en microempresas de Baja California. Problemas del Desarrollo. Revista Latinoamericana de Economía, pp. 173-194.

Organización Internacional del Trabajo (OIT). (2003). "17 Conferencia Internacional de Estadísticas de Trabajo". 2003 (págs. 1-92). Ginebra: OIT.

Osta Trestini, K. M. (2007). Desempleo e informalidad en América Latina: Definiendo políticas públicas para Venezuela. Revista Venezolana de Gerencia, pp. 262-278.

Parra Bernal, L. D. (2013). La empresarialidad informal como un reto de política económica: el caso de La Paz y El Alto, Bolivia. Estudios Gerenciales, pp. 485-491.

Parra, M., Catica, J. R., \& López, O. H. (2015). De emprendedores a empresarios, Historias que enseñan. Ibague: UT. 
Paz, J. (2013). Segmentación del mercado del trabajo en Argentina. Desarrollo y Sociedad, pp. 105-156.

Rodríguez Soto, J. R., \& Hernández Sanchez, J. (2013). Las Sociedades por Acciones Simplificadas, entre la flexibilidad y la formalización del emprendimiento empresarial. Revista Virtual Universidad Católica del Norte, pp. 123-136.

Rubio, M. V. (2014). El trabajo informal en Colombia e impacto en Latinoamérica. Observatorio Laboral Revista Venezolana, pp. 23-40.

Salazar Borrego, N. (2000). La Reforma Laboral en el marco de la Flexibilización. Gaceta Laboral, pp. 281-290.

Santa María, M., \& Rozo, S. (2009). Análisis cualitativo y cuantitativo de la informalidad empresarial en Colombia. Desarrollo y Sociedad, pp. 269-296.

Schneider, F. (2012). The Shadow Economy and Work in the Shadow: What Do We (Not) Know? Discussion Paper Series, pp. 4-73.

Schneider, F., \& Enste, D. (2000). Shadow Economies: Size, Causes, and Consequences. Journal of Economic Literature, pp. 77-114.

Schumpeter, J. (1934). The theory of economic development. Massachusetts- Cambridge: University Press.

Sociedades, S. d. (01 de noviembre de 2013). Informe Regional Bogotá 2009-2012. Recuperado de: http://www.supersociedades.gov.co/asuntos-economicos-y-contables/estudios-y-supervisionpor-ries gos/estudios-economicos-y-financieros/Documents/Regiones/Bogota\%202013.pdf.

Torresano, J. V., \& Díaz Chistiansen, S. (2014). El Fenómeno de la informalidad y su contribución al crecimiento económico: el caso de la ciudad de Guayaquil. Journal of Economics, Finance and Administrativa Science, pp. 90-97.

Townsend, F. (2000). Birth Order and Rebelliousness: Reconstructing the Research in Born to Rebel. Association for Politics and the Life Sciences, pp. 135-156. 